\title{
SYSTEMATIC GEOMETRIC IMAGE ERRORS OF VERY HIGH RESOLUTION OPTICAL SATELLITES
}

\author{
K. Jacobsen \\ Institute of Photogrammetry and GeoInformation, Leibniz University Hannover, Germany \\ jacobsen@ipi.uni-hannover.de
}

KEY WORDS: systematic image errors, validation, very high resolution optical satellites, height models, accuracy improvement

\begin{abstract}
:
Very high resolution optical satellites are imaging the object space by a combination of CCD-lines in one direction and by time, speed and satellite rotation in the other direction. The combination of the CCD-lines usually is known by pre-calibration. Remaining errors of the pre-calibration, also slightly depending upon the satellite movement and rotation, with few exceptions are usually small up to negligible. This may not be the case for the image component in the scan direction and the alignment of the line combinations they are controlled by giros and stellar cameras. Stellar cameras are compensating giro drifts, but their recording frequency is limited as well as in general the accuracy of the satellite view direction. In addition the satellites may show a jitter caused by the fast rotation from one pointed area to another. Not all giros are able to record the jitter frequency. A limited accuracy of the view direction is causing systematic image errors in relation to the used mathematical model of geometric reconstruction.

The systematic image errors can be determined theoretically by image orientation based on ground control points (GCPs), but usually not a satisfying number and distribution of GCPs is available. Another possibility is the analysis of the intersection of corresponding rays in a stereo model and an analysis of generated height models against reference height models. Here also free of charge available height models as the SRTM Digital Surface Model (DSM) or AW3D30 can be used. Several very high resolution satellite cameras have been analyzed; this includes images from WorldView-2, WorldView-4, Kompsat-3, Kompsat-1, Pleiades, Cartosat-1, ZY3, OrbView-3, QuickBird, IKONOS, ASTER, IRS-1C, SPOT, SPOT-5 HRS, EROS-B, IKONOS, QuickBird, OrbView and GeoEye but only results of the today more important satellites are shown in detail. For few satellites the systematic image errors can be ignored, but others require a correction which may be just a levelling of the DSM but also a higher degree of deformation up to a compensation of the satellite jitter effect.

The used method cannot be named as calibration due to variation from image to image, only the character and size of deformation is typical for the used special optical satellite, but it depends also upon the operating conditions as fast satellite rotation. Due to the very high number of reference points in a DSM the determination of systematic image errors is independent upon random errors and also high frequent jitter can be determined with a standard deviation down to 0.1 ground sampling distance (GSD) or even better.
\end{abstract}

\section{INTRODUCTION}

The geometry of optical satellite systems including the camera and the orientation of the single CCD-lines requires a satisfying calibration, attitude accuracy and time interval for recording attitude information (Wang et al. 2016), (Radhadevi et al. 2016), (Tong et al. 2014), (Jacobsen 2006a), (Jacobsen 2012), (Jacobsen 2017). The companies or governmental organizations, operating optical satellites, calibrate their systems in laboratory and in space (Dial 2003), (Valorge et al. 2003), but this is limited by the attitude quality which mostly is influenced by the situation of permanent satellite rotation for covering the planned ground area. Especially for stereoscopic coverage from the same orbit at least fast pitch rotations are required for the switch from forward view to the backward view. Such a rotation requires at first a strong angular acceleration, followed by negative angular acceleration which may cause a jitter during following imaging. The limited knowledge of the satellite attitude does not belong to the calibration it is individually for any scene. Only the character of the caused systematic image errors is similar for the optical satellite.

The image geometry can be determined by means of GCPs, but under operational conditions not enough GCPs are available. In addition GCPs for satellite camera calibration must be large symmetric objects for reaching the required pointing accuracy.
Most often used corner points shift their position depending upon the object illumination. Digital Globe used an aerial block, flown with a Leica DMC, as ground control reference leading to 15502 indirect GCPs for the verification of the WorldView-4 calibration (Comp 2017). Of course this is also a higher effort not possible for everyone.

In case of a block of space images, systematic errors can be determined by means of the over-determination (d' Angelo et al. 2013), but such blocks are only used by large organizations. The satellite roll differences of a stereo coverage can be determined by means of y-parallaxes of intersections for ground coordinate computation. Problems of the satellite pitch are influencing the point heights, so it is possible to determine it by comparison of a generated height model with a reference height model. This reference height model even may have a lower accuracy as the determined DSM due to the fact, that a very high number of points are included in the DSM, nearly eliminating the random errors of the reference DSM. The free of charge available height models as from SRTM and AW3D30 are not based on just one data set, in case of SRTM at least two data sets are used, in addition with interferometric synthetic aperture radar the distance and not the attitude is important for georeference, so model deformations don't have to be expected. AW3D30 is using all available ALOS PRISM images, reducing the probability of systematic errors of the reference height model. Yaw errors are not as critical as roll and pitch errors due 
to only slow satellite yaw rotation. A yaw discrepancy tilts a generated height model around the base line.

Systematic image errors or caused height model orientation errors and deformations may be different from model to model; nevertheless they are reducing the accuracy and can be at least partially compensated by using reference height models. Even only a validation is useful for a better estimation of the achieved quality of mapping results.

\section{BASIC IMAGE GEOMETRY}

Systematic image errors of satellite images are the differences of the image geometry against the mathematical model used for image orientation. Image orientation is the determination of the relation between image and ground coordinates. As basic mathematical model a reconstruction of the imaging geometry is used, which is based on the geometry of the joint combination of the CCD-elements to a homogenous CCD-line. For example for the panchromatic channel WorldView-2 has a combination of 60 staggered CCD-elements in the focal plane (figure 1). This combination causes that even and odd original CCD-lines, finally used for the combination of a homogenous line, are taken at a different instant. This time delay and the physical CCD-line combination are respected by the internal calibration of the satellite organization - the standard image user has no information about calibration and also no access to the original sub-images of the individual CCD-sensors. In addition these are not single CCD-lines, for the extension of the extremely short imaging time for one pixel, time delay and integration (TDI) sensors are used, based on small CCD-arrays, shifting the charge from one CCD-line to the next and accumulating over the lines of a TDI-sensor the charge. The use of TDI-sensors is not influencing the geometric image behaviour.

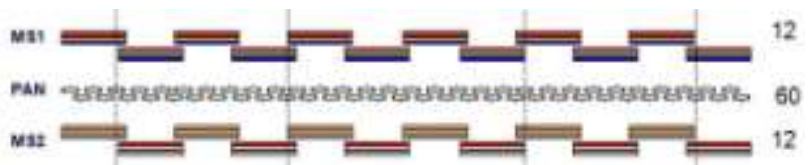

Figure 1. Focal plane arrangement of WorldView-2 (Digital Globe)

Usually the colour information has a 4 times lower resolution, used for pan-sharpening. The colour bands are fitted to the panchromatic information, so the image geometry is dominated by the panchromatic band.

Only the first high resolution digital space sensors have not been flexible satellites. Today we have body pointing flexible satellites, able to rotate very fast. Also during imaging the satellite rotates from one line to the next.

If the sample rate does not correspond to the satellite speed, we have a so called asynchronous imaging (figure 2). In this case the satellite is changing the view direction permanently. In figure 2 the distance A corresponds to the base length of a not rotating satellite, while the distance $\mathrm{B}$ corresponds to the used distance in the orbit for generating a scene. The relation $\mathrm{B} / \mathrm{A}$ is named as slow down factor. For example EROS-B was not equipped with TDI-sensors and had to use a slow down factor 3.30 for having enough time for imaging. Today most satellite sensors have a slow down factor close to 1.0 or in case of WorldView-1 even 0.59, allowing a fast imaging. But not only for asynchronous imaging the satellite is rotating during imaging; the flexible optical satellites can take the covered scene area in any direction - usually in the map projection north-south direction but it can be also in the south-north or in any other direction.

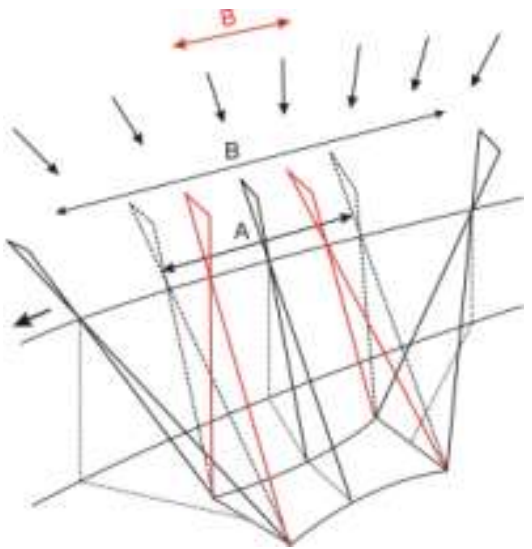

Figure 2. Asynchronous imaging

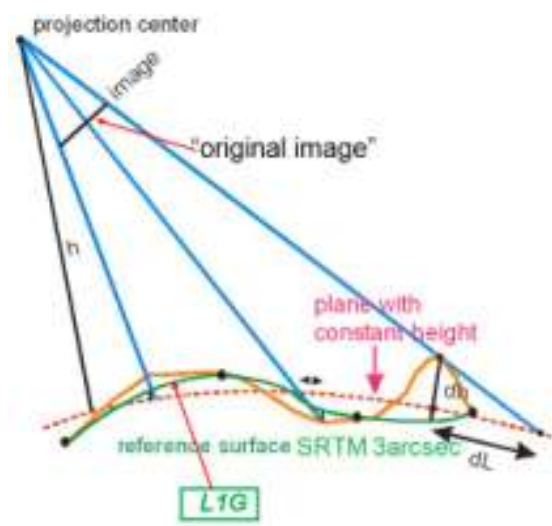

Figure 3. Satellite image types

Optical satellite images are distributed with different geometry. There is no standard of the naming. "Original images" (figure 3), also named Level 1A, L1R or Basic, are the joint images of the sub-CCD-elements using the inner orientation calibration and radiometric improvement. This is the lowest level of preprocessing for the user. The projection of the image in the map projection to a plane with constant height (ortho image with $\mathrm{Z}=$ constant) is named Level $2 \mathrm{~A}$, OR Standard or Geo. The next step is an ortho image with a specified height model, named as Level 3, L1G (Kompsat) or Standard in case of QuickBird.

In general no different image accuracy exists between Level 1A or $2 \mathrm{~A}$ images. This is not the case for L1G images from Kompsat- 3 where the influence of the SRTM 3 arcsec height model could not be respected correctly, while the L1R image of the same scene was delivering satisfying results (Jacobsen 2018).

\section{IMAGE ORIENTATION}

As mentioned above, the image orientation determines the relation between image and ground coordinates. The imaging geometry can be reconstructed based on the information in the image header data, but today dominates the replacement model by rational polynomial coefficients (RPC) (Jacobsen 2003). The $\mathrm{RPC}$-orientation is based on the direct sensor orientation of the satellite. Due to the limitation of the attitude information it has to be improved with GCPs by the bias corrected RPC solution. As bias correction at least shifts in $\mathrm{X}$ and $\mathrm{Y}$ are required, but usually a 2-dimensional affine transformation has to be used. In some cases also an additional improvement of the view direction by one coefficient for X- and one for Y-direction has to be used. Simplified models as the 3D-affine transformation 
(Hanley et al. 2002) or the extended 3D-affine transformation (Jacobsen 2006b) even can be used for small field of view images and a height variation of GCPs.

\section{SYSTEMATIC IMAGE ERRORS}

The influence of systematic image errors of a satellite stereo pair can be determined with the over-determination in the yparallax by intersection for the ground location. In addition the systematic height model deformation can be compared with a reference height model. Of course this does not allow a calibration, but a validation. The calibration in orbit direction also with a high number of ground control points is not totally possible due to the influence of the attitude being different from stereo model to stereo model, depending upon the gyros, supported by star sensors, having the limiting influence to direct geo-reference. The images are not only taken in north-south direction, stereo models are often scanned across orbit direction, or for scenes related to the ground coordinate system, in east-west direction. That means the influence of systematic yparallax may be seen also in east-west direction.

\subsection{Analysis of $y$-parallaxes}

The average y-parallaxes are computed for equal distances as function of the ground $\mathrm{X}$ - and ground $\mathrm{Y}$-coordinates by the program for computing the ground coordinates via intersection, usually done for digital surface models (DSM). The high number of matched points in the range above 10 million points eliminates random effects. The analysis of the reason for systematic Y-parallaxes has to respect the scan direction, so a satellite jitter will be shown in the scan direction which may be also across orbit.

A typical example for a satellite jitter is shown by the systematic errors of the WorldView-2 (WV-2) stereo models taken at the Black Sea cost in Turkey. Three stereo models located in eastwest direction side by side have been imaged from one orbit. This required a fast rotation of the WV-2 satellite pointing at first forward to the three areas and after this backwards. Only 10 seconds are between the scan starts of the images. Together with the movement from one to the next scene, there was not enough time after fast rotation to avoid a jitter.

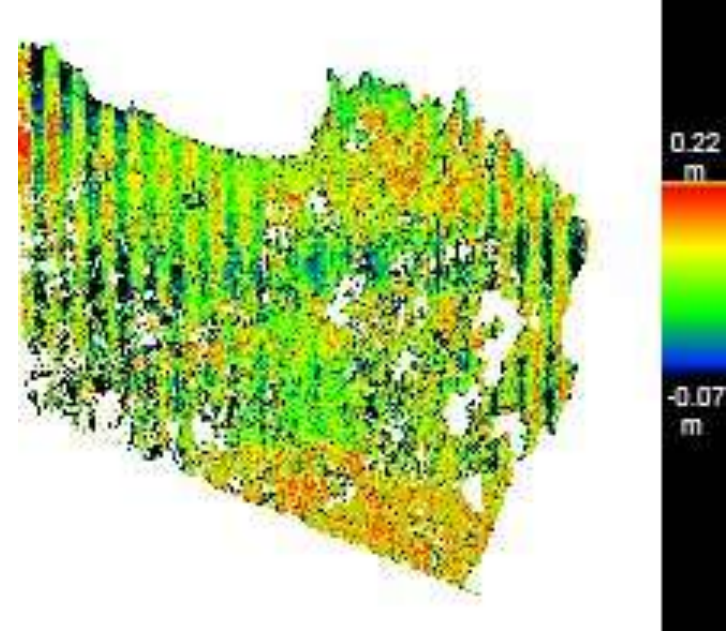

Figure 4. Averaged y-parallaxes of a WV-2 stereo model

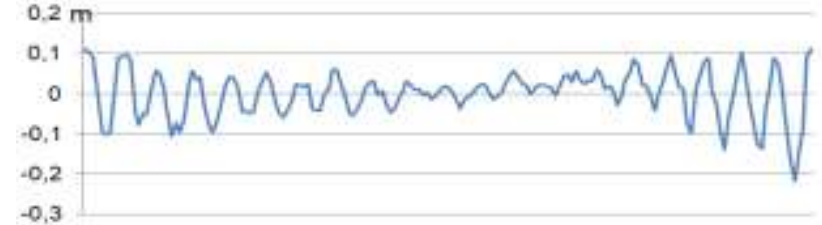

Figure 5. Averaged WV-2 y-parallax over 16km in east-west

Figures 4 and 5 are demonstration the satellite jitter. Nevertheless the y-parallaxes are limited to the range of $+/$ $0.1 \mathrm{~m}$, corresponding to 0.2 pixels, in the average, so they are not a mayor problem for the object point coordinates. Mainly the image quality is influenced by the high frequent jitter. In the north-south direction (Figure 6), which corresponds to the CCD-line direction, no jitter effect is expected and not visible. The root mean square differences are limited here to $6 \mathrm{~cm}$ or 0.13 pixels. Even if the jitter would be known, it could not be respected in the third order polynomials of the RPC.

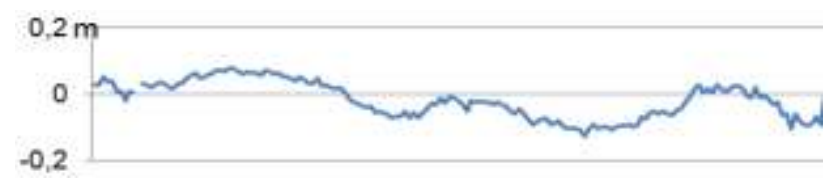

Figure 6. Averaged WV-2 y-parallax over $8 \mathrm{~km}$ in north-south

A WorldView-4 (WV-4) stereo pair, covering $8 \mathrm{~km}$ times $21 \mathrm{~km}$, and scanned in north-south direction, does not indicate periodic errors in east-west direction (Figure7), only in the north south direction a small scintillation can be seen (Figures 8). Nevertheless the effective image quality, determined by edge analysis (Jacobsen et al. 2016), corresponds to $40 \mathrm{~cm}$ GSD instead of the nominal $31 \mathrm{~cm}$. The root mean square of the systematic y-parallax in east-west direction is $5 \mathrm{~cm}$, corresponding to 0.2 pixels and in north-south direction $8 \mathrm{~cm}$ or 0.26 pixels.

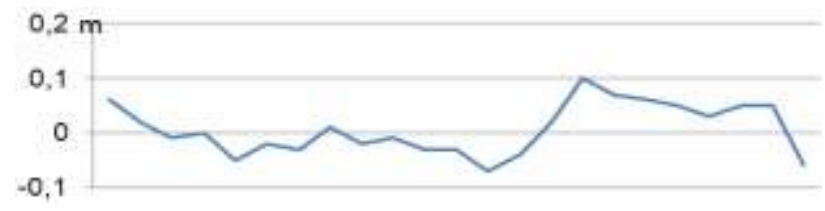

Figure 7. Averaged WV-4 y-parallax over $3 \mathrm{~km}$ in east-west

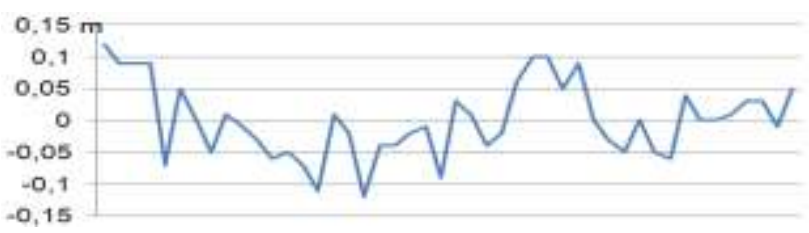

Figure 8. Averaged WV-4 y-parallax over $19 \mathrm{~km}$ in north-south

The averaged y-parallax profiles of a Kompsat-3 stereo pair (figures 10 and 11) does not indicate a jitter. The root mean square of the averaged y-parallaxes is $9 \mathrm{~cm}$ in east-west and $7 \mathrm{~cm}$ in north-south direction, corresponding to approximately 0.16 pixels. This is not far away from WorldView-4. The area distribution of the averaged y-parallaxes (figure 9), based on 20 million image points, is quite different as for WorldView-2 - no jitter is indicated; only a homogenous distribution can be seen, corresponding to a model deformation. 


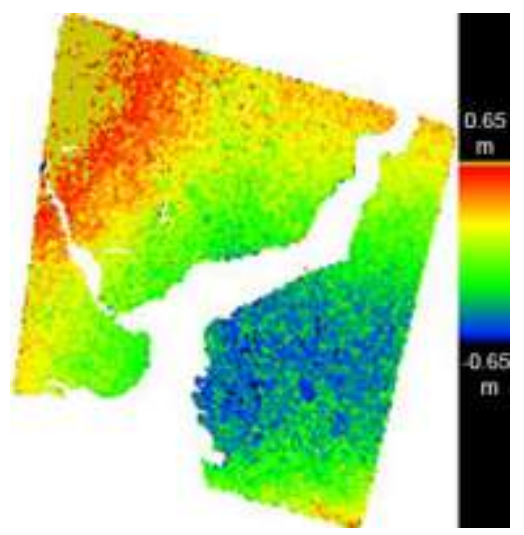

Figure 9. Averaged y-parallaxes, Kompsat-3 stereo model

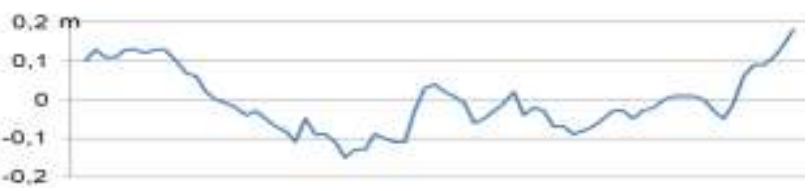

Figure 10. Averaged Kompsat-3 y-parallax over $8 \mathrm{~km}$ in E-W

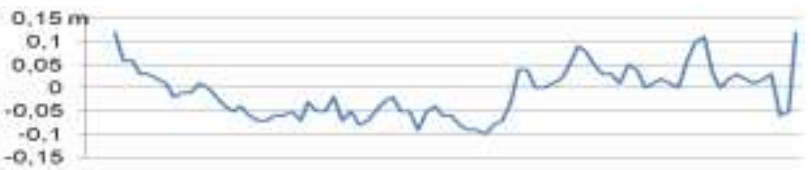

Figure 11. Averaged Kompsat-3 y-parallax over $8 \mathrm{~km}$ in N-S

The analysis of a Pleiades stereo model based on 36 million height points (figures 12 - 14) shows excellent results in relation to the other data sets. The area distribution of the yparallaxes (figure 12) is just reaching $-11 \mathrm{~cm}$ to $10 \mathrm{~cm}$. The $\mathrm{y}-$ parallax profile in east-west direction, being dependent upon the CCD-line, has just a root mean square size of $0.5 \mathrm{~cm}$ or 0.01 pixels and in the north-south direction $1.8 \mathrm{~cm}$ or 0.04 pixels. That means the inner orientation of the Pleiades camera is very good as well as the attitude control indicated by the y-parallax profile in north-south direction.

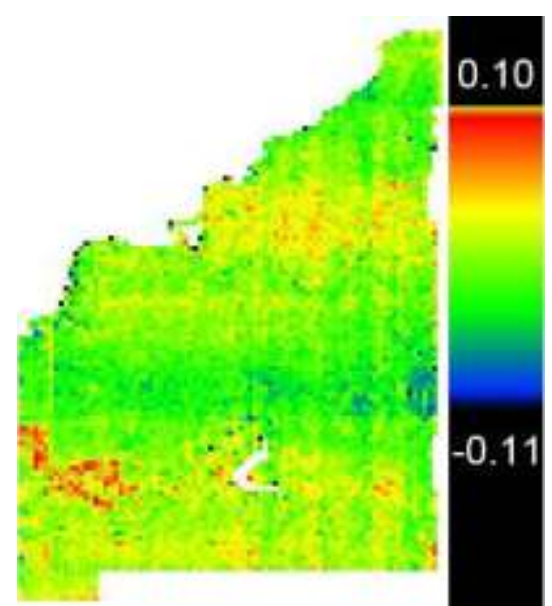

Figure 12. Averaged y-parallaxes, Pleiades stereo model

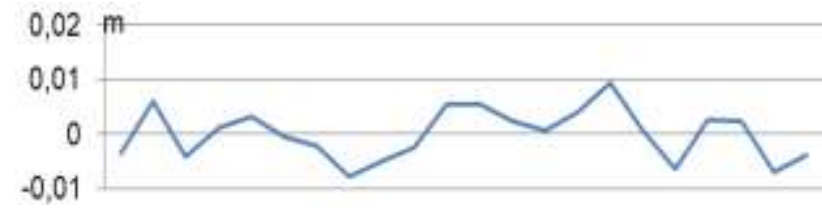

Figure 13. Averaged Pleiades y-parallax over $8 \mathrm{~km}$ in east-west

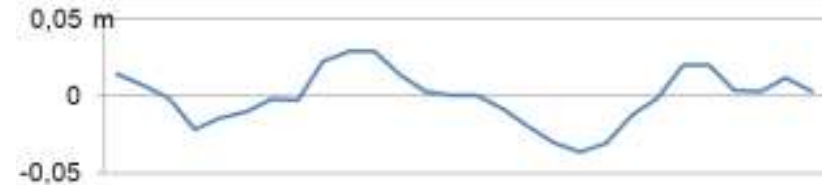

Figure 14. Averaged Pleiades y-parallax over 8km, north-south

In the ISPRS Cartosat- 1 test area Mausanne the area distributed systematic y-parallax (figure 15) shows in north-south direction some periodic effects, being more clear with the north-south profiles (figure 17). This indicates also a satellite jitter, but with lower frequency as shown for WorldView-2. The jitter frequency depends upon the size of the satellite and the behaviour of the stabilization devices. Cartosat- 1 is a stereo satellite with a GSD of $2.5 \mathrm{~m}$. Stereo satellites do not have fast angular rotations as the flexible satellites; nevertheless their orientation also has to be corrected which may cause a limited jitter. In other investigated Cartosat- 1 data sets no jitter effect is available. Figure 15 shows a scene rotation, not presented by the y-parallax profiles due to the levelling of the shown profiles. In east-west direction, dominated by the CCD-line, a stronger tilt on the left hand side of the y-parallax profile (figure 16) by $1 \mathrm{~m}$, corresponding to 0.4 pixels is shown. A similar behaviour, but with another form, can be seen also in other Cartosat-1 stereo models. Despite of this, the weighted root mean square differences of the averaged y-parallaxes in the test area Mausanne are $18 \mathrm{~cm}$ respectively $14 \mathrm{~cm}$ corresponding to 0.07 pixels which is even smaller as for other satellites.

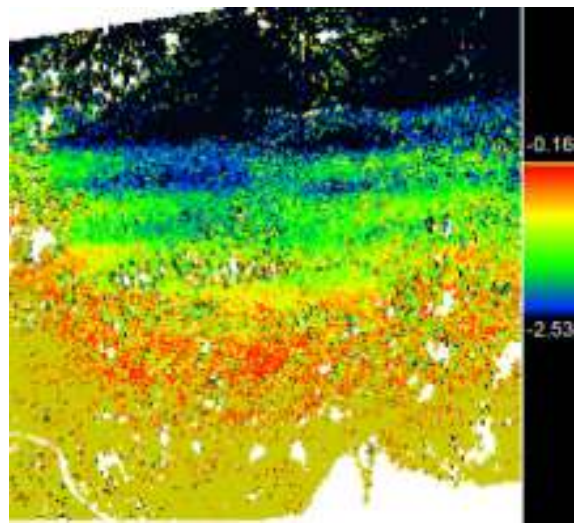

Figure 15. Averaged y-parallaxes, Cartosat-1 stereo model

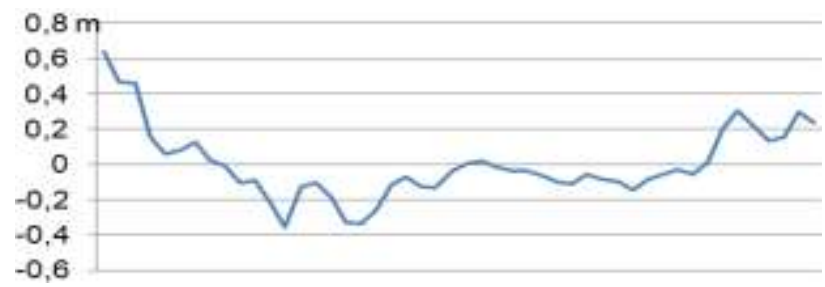

Figure 16. Averaged Cartosat-1 y-parallax over $34 \mathrm{~km}$ east-west

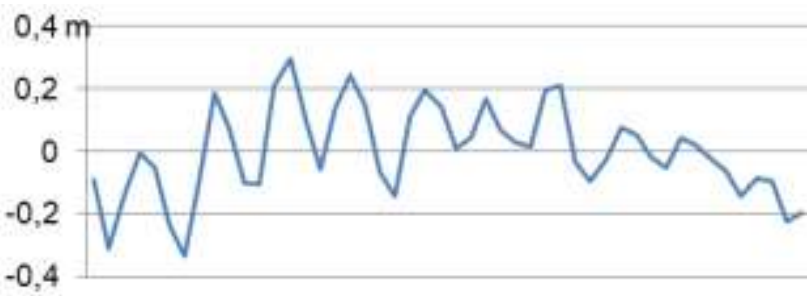

Figure 17. Averaged Cartosat-1 y-parallax over $34 \mathrm{~km}$ in N-S 


\subsection{Analysis of height models}

The comparison of a height model determined with a satellite stereo pair against a reference height model allows also a detailed verification of the stereo pair geometry. The verification depends upon the type of reference. By matching of optical satellite images digital surface models (DSM) are generated, but the surface is not the same as with a DSM from LiDAR. Especially in areas with vegetation there is a clear difference between both types of DSM. If systematic height differences between a satellite DSM and a LiDAR DSM shall be analyzed, vegetation areas have to be filtered out. If free of charge available large area covering DSM shall be used, as from SRTM, ASTER GDEM or AW3D30, this problem does not exist. The SRTM DSM is based on the radar C-band; this describes also the visible surface. Only with long wavelength radar as L-band, the vegetation, as also forest, can be penetrated, but such a nearly worldwide height model is not yet available. ASTER GDEM and AW3D30 are based on optical images, describing the surface similar as high resolution optical images. Nevertheless the visible surface of vegetation depends upon the annual growing season. In cultivated forest areas, as we have it in Europe, the forest is growing up until the clear cut. So it is better to eliminate such vegetation areas if we like to analyze and correct systematic height model errors caused by systematic image errors in the base direction corresponding to satellite pitch.

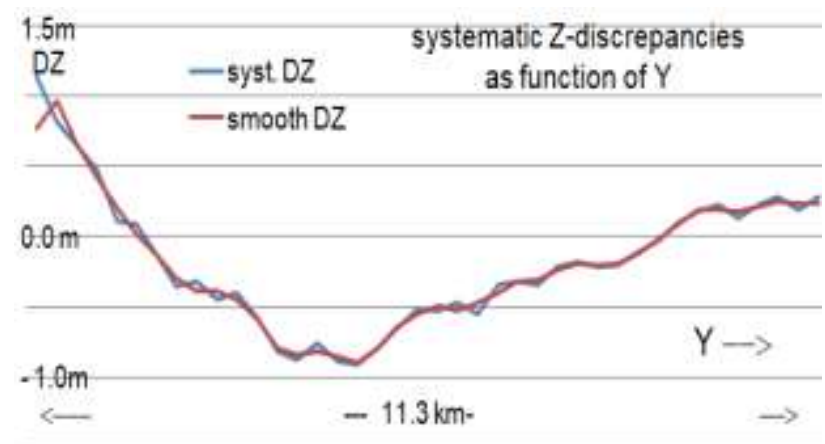

Figure 18. Systematic Z-discrepancies of Kompsat-3 DSM as function of $Y$

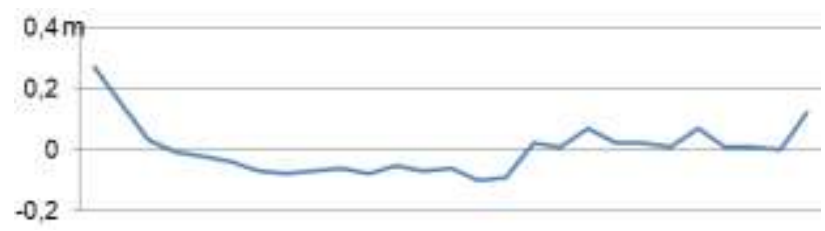

Figure 19. Systematic Z-discrepancies of Kompsat-3 DSM as function of X; profile length $=34 \mathrm{~km}$

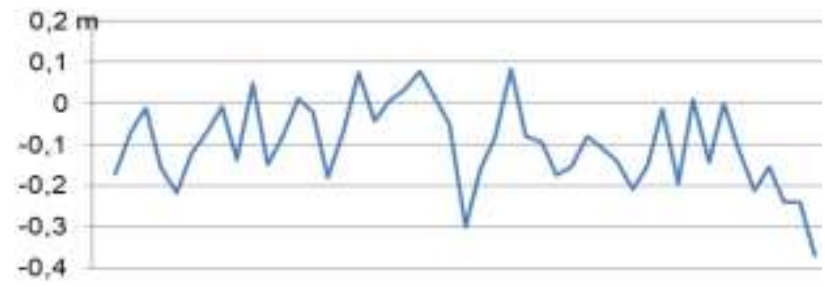

Figure 20. Systematic Z-discrepancies of Kompsat-3 DSM as function of $\mathrm{Y}$ after improvement with AW3D30

Figure 18 shows the systematic height differences of a Kompsat-3 DSM over Istanbul against an airborne LiDAR DSM. The Z-discrepancies are averaged as function of their ground coordinates $\mathrm{Y}$, in this case in $50 \mathrm{Y}$-coordinate-groups. The red line is a smoothened function which can be used for the improvement of the DSM. Kompsat-3 has $71 \mathrm{~cm}$ GSD for nadir view, in this case due to the inclined view in the average $80 \mathrm{~cm}$ GSD. The image quality corresponds to the nominal resolution. With the GSD and the base to height relation of $1: 1.2$ the systematic height differences in the Y-direction of $+1 \mathrm{~m}$ down to $-0.9 \mathrm{~m}$ are not acceptable. The systematic $\mathrm{Z}$-differences in the $\mathrm{X}$ direction have a root mean square size of approximately $12 \mathrm{~cm}$ (figure 19). This can be seen as remaining effect of not eliminated vegetation or as noise; it is unimportant in relation to the expected Z-accuracy. After improvement of the Kompsat-3 DSM based on the nearly worldwide AW3D30, the systematic Z-differences are reduced also in the Y-direction in the root mean square to $9 \mathrm{~cm}$ (figure 20). This corresponds to figure 17 . Again - the systematic Z-differences in Y-direction are depending upon the pitch attitude, while in the $\mathrm{X}$-direction it is dominated by the CCD-line. If the calibration of the CCD-line connection is satisfying, no geometric problems in the $\mathrm{X}$ direction are expected if the scene is scanned in north-south direction.

As also shown in (Jacobsen 2017) instead of a reference height model from aerial LiDAR or from aerial photogrammetry also the nearly worldwide height models from SRTM or AW3D30 can be used for a determination and compensation of systematic height models. There is only a problem of levelling the reference height models which is possible based on the ground control points used for bias corrected RPC-orientation.

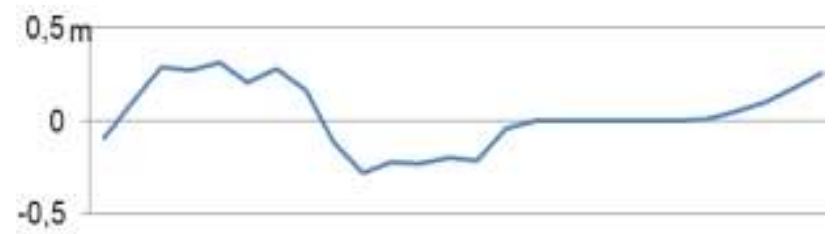

Figure 21. Systematic Z-discrepancies of WorldView-4 DSM as function of $Y$

In an overlapping area of Istanbul, also a WorldView-4 stereo model was analyzed. WorldView- 4 has $31 \mathrm{~cm}$ GSD and the stereo model a base to height relation of $1: 1.2$. The systematic Z-differences against an airborne LiDAR DSM in the root mean square are reaching $30 \mathrm{~cm}$ (figure 21 ) but only in the sub-areas with lower point density. The root mean square of the systematic Z-differences, weighted for the number of observations in the groups, is just $13 \mathrm{~cm}$ and this is not an important size.

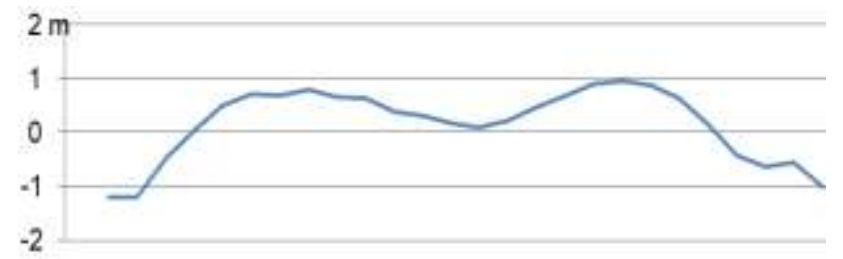

Figure 22. Systematic Z-discrepancies of Zyuan-3 DSM as function of Y; profile length $=55 \mathrm{~km}$, DSM levelled

The characteristic of the systematic height differences are different for any sensor and also the individual data set. In the Y-direction the satellite pitch changes are dominating the height discrepancies as it is visible in figure 22 for Zyuan-3, showing a low period rotation which should be used for a DSM improvement due to the weighted root mean square influence of $0.93 \mathrm{~m}$ 


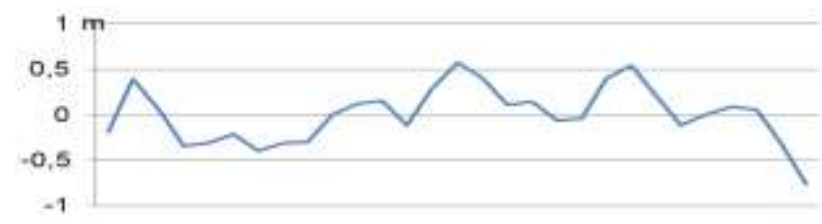

Figure 23. Systematic Z-discrepancies of SPOT-5 HRS DSM as function of $Y$; profile length $=5 \mathrm{~km}$

In SPOT-5 HRS height models as shown in figure 23 for the test area GARS no important systematic errors could be seen. The root mean square systematic error is just reaching $0.40 \mathrm{~m}$ or 0.08 pixels.

\section{CONCLUSION}

Due to the high number of tie points for the determination of height models the analysis of the systematic y-parallaxes is very sensitive also for small systematic image errors even below 0.1 pixels. With the y-parallaxes no camera calibration is possible, but it can be used for verification. There is a clear difference for discrepancies in scan direction and across to it. Across the scan direction the image geometry depends upon the CCD-line combination, while in the scan direction the accuracy depends upon the attitude which may be influenced by a satellite jitter. The determined systematic effects usually do not exceed 0.2 pixels; only in the case of one Cartosat- 1 stereo pair it reached locally 0.4 pixels, but this was also the case for Ziyuan-3 (Jacobsen 2017) showing a not very homogenous systematic yparallax profile in the orbit direction. The highest accuracy was reached with a Pleiades stereo scene having systematic effects clearly below 0.1 pixels. Of course the determination of systematic y-parallaxes depends upon the usually used bias corrected RPC-orientation. With the third order polynomials of RPCs the high frequency of a satellite jitter cannot expressed even if it has been recorded.

Of course the systematic y-parallaxes cannot be used for the improvement of the achieved results; they only indicate some geometric problems which may be available also in the other coordinate components. This is different for the systematic height model errors; they can be used after high pass filtering directly for the improvement of the generated height models. Here a support by the free available nearly worldwide height models is possible. AW3D30 is proposed; it is newer as the SRTM DSM and in addition slightly more accurate (Bayburt et al.). The requirement of such an a posterior height model improvement depends upon the used optical satellite, for some it is not required, for some in any case and for some it may be. So at least verification for systematic height model errors is recommended.

\section{REFERENCES}

d'Angelo, P., 2013: Automatic orientation of large multitemporal satellite image blocks: Proceedings of International Symposium on Satellite Mapping Technology and Application 2013

Bayburt, S., Kurtak, A.B., Büyüksalih, G., Jacobsen, K., 2017: Geometric accuracy analysis of WorldDEM in relation to AW3D30, SRTM and ASTER GDEM2, ISPRS Hannover Workshop 2017, ISPRS Archives - Volume XLII-1/W1, pp 211-217
Comp, C., Mulawa, D., Clarke, B., Updike, T., 2017: JACIE 2017

Dial, G., 2003: Test ranges for metric calibration and validation of satellite imaging systems, Workshop on Radiometric and Geometric Calibration, Gulfport, 2003, on CD

Hanley, H.B., Yamakawa. T., Fraser, C.S., 2002: Sensor orientation for high resolution imagery, ISPRS Archives XXXIV, part 1, paper 90

Jacobsen, K., 2003: Geometric potential of IKONOS- and QuickBird-images, in D. Fritsch (Ed.) Photogrammetric Weeks '03, pp 101-110, Wichmann Verlag ISBN 3-87907-397-X and GIS Geo-Informations-Systeme 9/2003, pp33 - 39

Jacobsen, K., 2006a: Comparison of image orientation by IKONOS, QuickBird and OrbView-3, EARSeL 2006, Warsaw, in "New Developments and Challenges in Remote Sensing", edited by Z. Bochenek, Millpress, Rotterdam 2007 ISBN 97890-5966-053-3, pp $667-676$

Jacobsen, K., 2006b: Calibration of optical satellite sensors, EuroCOW, Castelldefels 2006

Jacobsen, K., 2012: Calibration, sensor models and orientation pp 127-172 in: Dowman, I., Jacobsen, K., Konecny, G., Sandau R., High Resolution Optical Satellite Imagery, Whittles Publishing ISBN 978-184995-046-6

Jacobsen, K., Topan, H., Cam, A., Özendi, M., Oruc,M., 2016: Image quality assessment of Pleiades-1A triplet bundle and pansharpened images, PFG 3/2016, pp 75-86

Jacobsen, K., 2017:Problems and limitations of satellite image orientation for determination of height models, ISPRS Hannover Workshop 2017, ISPRS Archives - Volume XLII1/W1, pp 257- 264

Jacobsen, K., Sefercik, U., 2018: Dense Matching mit WorldView-4 und Kompsat-3 Bildern, DGPF Jahrestagung 2018

Radhadevi, P.V., Solanki, S. S. , Akilan, A., Jyothi, M. V., Nagasubramanian,V., 2016: Performance assessment and geometric calibration of Resourcesat-2, Int. Arch. Photogramm. Remote Sens. Spatial Inf. Sci., XLI-B1, 237-244, 2016

Srinivasan, T.P., B. Islam, Sanjay K. Singh, B. Gopala Krishna, P. K. Srivastava, 2008: In-flight geometric calibration - an experiment with Cartosat-1 and Cartosat-2, ISPRS Archives, Volume XXXVII-B1, pp 83-88

Tong, X., Ye, Z., Xu, Y., Tang X., Liu, S., Li, L., Xie, H., Wang, F., Li, T. And Hong Z., 2014: Framework of jitter detection and compensation for high resolution satellites, Remote Sensing 2014, 6, 3944-3964

Valorge, C., Meygret, A., Lebègue, L., Henry, P., Bouillon, A., Gachet, R., Breton, E., Léger, D.,Viallefont, F., 2003 : 40 years of experience with SPOT in-flight calibration, Workshop on Radiometric and Geometric Calibration, Gulfport, 2003, on CD

Wang, M., Cheng, Y., Long, X., Yang, B., 2016: On-orbit calibration approach for high-resolution geostationary optical satellite GaoFen-4, ISPRS Archives - Volume XLI-B1, 2016, pp 389-394 